\title{
Iron and Mechanisms of Neurotoxicity
}

\author{
Gabriela A. Salvador, Romina M. Uranga, and Norma M. Giusto \\ Instituto de Investigaciones Bioquímicas Bahía Blanca, Universidad Nacional del Sur y Consejo Nacional de Investigaciones Científicas \\ y Técnicas, 8000 Bahía Blanca, Argentina
}

Correspondence should be addressed to Gabriela A. Salvador, salvador@criba.edu.ar

Received 13 September 2010; Accepted 10 November 2010

Academic Editor: Anthony R. White

Copyright ( $) 2011$ Gabriela A. Salvador et al. This is an open access article distributed under the Creative Commons Attribution License, which permits unrestricted use, distribution, and reproduction in any medium, provided the original work is properly cited.

\begin{abstract}
The accumulation of transition metals (e.g., copper, zinc, and iron) and the dysregulation of their metabolism are a hallmark in the pathogenesis of several neurodegenerative diseases. This paper will be focused on the mechanism of neurotoxicity mediated by iron. This metal progressively accumulates in the brain both during normal aging and neurodegenerative processes. High iron concentrations in the brain have been consistently observed in Alzheimer's (AD) and Parkinson's (PD) diseases. In this connection, metalloneurobiology has become extremely important in establishing the role of iron in the onset and progression of neurodegenerative diseases. Neurons have developed several protective mechanisms against oxidative stress, among them, the activation of cellular signaling pathways. The final response will depend on the identity, intensity, and persistence of the oxidative insult. The characterization of the mechanisms mediating the effects of iron-induced increase in neuronal dysfunction and death is central to understanding the pathology of a number of neurodegenerative disorders.
\end{abstract}

\section{Introduction}

The so-called "biometals" (e.g., iron, copper, or zinc) are known to play a fundamental role in numerous essential metabolic processes, thus being considered as essential for life. Metal ion homeostasis is maintained through highly regulated mechanisms of uptake, storage, and secretion [1]. A specific set of transporters functions in each cellular compartment to provide a strict balance of transport activities across their membranes. Nonbound copper and iron are potentially harmful mainly due to their redox activities. Normally, under healthy conditions, these metal ions are bound to ligands (e.g., transferrin, ceruloplasmin), and they are not found as free species. However, the release of free ionic or exchangeable zinc and copper has been reported in the synaptic cleft. In addition, zinc is also being increasingly involved in several cellular reactions like calcium, and it has been proposed as a new class of second messenger.

A loss or an abnormal metal homeostasis might cause cellular death or severe dysfunction, and it has been recognized as a triggering factor for different neurodegenerative disorders such as Alzheimer's (AD), Parkinson's (PD), and
Huntington's (HD) diseases as well as amyotrophic lateral sclerosis (ALS) [2-6]. Although the etiology of these diseases is still largely unknown, oxidative damage mediated by metals has been thought to be a significant contributor since metals such as iron, aluminum, zinc, and copper have been observed to be dysregulated and/or increased in $\mathrm{AD}$ brains and prone to generate a pro-oxidative environment [7-11]. Taking into account the great amount of information regarding the role of transition metals in cell biology, this paper will be mainly focused on the role of iron in neurodegeneration.

Loss of iron may cause neurological disease, and, in opposition, its accumulation or abnormal interaction with cellular components such as proteins, lipids, or nucleic acids may also contribute to neurodegenerative disorders. The intracellular pool of free iron, the labile iron pool (LIP), has been well established to modulate the expression of various proteins, including the amyloid precursor protein (APP) $[12,13]$.

In the brain, the movement of metals across the bloodbrain barrier is highly regulated, and there is no passive flux of metals from the circulation to the brain $[1,14]$. 
While iron, copper, and zinc are being increasingly implicated in interactions with the major protein components of neurodegenerative diseases, this is not merely due to increased (e.g., toxicological) exposure to metals but rather because of a breakdown in the homeostatic mechanisms that compartmentalize and regulate metals [7].

The ability of iron to accept and donate electrons can lead to the formation of reactive nitrogen and oxygen species (the latter named "ROS" in this paper) which may trigger the oxidative attack of tissue components, therefore contributing to disease and perhaps aging itself $[15,16]$. Increasing age is the main risk factor associated with the appearance of neurodegenerative diseases. Several studies in animals and humans have reported a rise in brain iron as a function of ageing $[17,18]$. The vulnerability of the brain to abnormal iron regulation has been demonstrated by the relationship between the failure of ferroxidases, ceruloplasmin $[19,20]$, ferritin $[18,21,22]$, and frataxin $[23,24]$, iron accumulation (IA), and the onset of neurodegenerative diseases.

\section{Iron Accumulation in Neurodegenerative Diseases}

The accumulation of transition metals in the nervous system is a common observation in different neurodegenerative diseases that support a role of metals in these disorders $[25,26]$. Particularly, iron homeostasis has shown to be altered [26, 27]. Excessive iron deposition has been reported to occur in the central nervous system (CNS) in a number of neurodegenerative pathologies such as $\mathrm{AD}, \mathrm{PD}, \mathrm{ALS}$, and neuroferritinopathies, among others [4, 28-33].

ALS is a neurodegenerative disorder characterized by progressive paralysis of skeletal muscles and degeneration of motor neurons in the spinal cord, brainstem, and cortex. High levels of iron in the CNS of both familial and sporadic forms of ALS have been reported [32, 34, 35]. However, neither the mechanisms underlying iron accumulation nor its complete role in the pathogenesis of the disease are clear.

Neuroferritinopathies, like neurodegeneration with brain iron accumulation (NBIA), are defined as extrapyramidal disorders [33] characterized by radiographic evidence of focal iron accumulation in the brain [36]. These diseases are progressive movement disorders caused by nucleotide insertions in exon 4 of the ferritin light chain gene [37]. These patients show low levels of serum ferritin [22] and abundant spherical inclusions in the brain, skin, kidney, liver, and muscle [33] that are positive for iron, ferritin, and ubiquitin staining [38].

Moreover, abnormal accumulation of iron is also considered to be involved in the pathogenesis of myelin diseases such as multiple sclerosis (MS). Histochemical studies have shown that abnormal iron deposits are observed in reactive microglia, axons, neurons, and oligodendrocytes in patients with MS $[39,40]$. Indeed, ferritin levels are increased in the CNS of mice with experimental autoimmune encephalomyelitis, an animal model of MS [41], and in the cerebrospinal fluid of MS patients [42, 43]. Since the synthesis of ferritin can reduce toxic ferrous iron $\left(\mathrm{Fe}^{2+}\right)$, the elevated level of ferritin in autoimmune encephalomyelitis mice and MS patients is considered to be cytoprotective [40].

Although iron has been demonstrated to have a potential role in many diseases of the CNS, this paper will focus mainly on the information regarding the role of iron in $\mathrm{AD}$. It is well known that transition metals provoke oxidative stress by generating ROS through the Fenton reaction, thus causing brain lipid peroxidation [44] and protein oxidation $[45,46]$. Interestingly, not only has iron been involved in lipid and protein oxidation but also in DNA damage. It has been shown that iron is able to oxidize DNA bases, and it has been suggested that the accumulation of this transition metal observed in some neurodegenerative disorders could act by both increasing oxidative genome damage and also preventing its repair [47]. Iron itself has been related to neurotoxicity, and its accumulation, mainly in the hippocampus and cortex, has been observed to occur before $\mathrm{AD}$ lesions are detectable. Moreover, it has been also demonstrated to accumulate both in $\mathrm{AD}$ senile plaques [48] and in amyloid deposits in A $\beta$ PP2576 transgenic mouse model of $A D$ [49]. Interestingly, $A \beta$ insoluble aggregates have been shown to be dissolved by metal chelators [50].

Oxidative stress is considered to be the earliest change in the pathogenesis of $\mathrm{AD}$, and high levels of oxidative stress have been demonstrated to occur in the clinical precursor of $\mathrm{AD}$, known as mild cognitive impairment (MCI) [51, 52]. Coincidently, increased iron levels were found both in the cortex and cerebellum from the preclinical $\mathrm{AD} / \mathrm{MCI}$ cases. Moreover, iron concentrations have been found to be increased in the bilateral hippocampus, parietal cortex, frontal white matter, putamen, caudate nucleus, thalamus, red nucleus, substantia nigra, and dentate nucleus subregions of patients with diagnosed $\mathrm{AD}$ and in normal elderly patients $[53,54]$. It is important to note that these brain iron concentrations, particularly those in the parietal cortex at the early stages of $\mathrm{AD}$, have been found to positively correlate with the severity of patients' cognitive impairment [53]. Although extensive evidence links the dysregulation of iron homeostasis and $\mathrm{AD}$, relatively little is known about the resulting forms of iron that accumulate in the brain. Numerous techniques have been developed in order to characterize, locate, and quantify iron species and ironcontaining compounds in AD. For example, the use of iron fluorescence together with synchrotron X-ray absorption spectroscopy showed in situ iron accumulations containing high concentrations of ferritin and magnetite in $\mathrm{AD}$ brain tissue sections [55].

Alterations in iron metabolism with age have been described, and they may involve iron uptake and release, storage, and intracellular metabolism [56-59]. Although some issues remain unclear, it is well known that the dyshomeostasis of brain iron metabolism is one of the initial events that trigger neuronal death in some neurodegenerative disorders [60-63]. Existing evidence shows that these mechanisms may well be altered by the ageing process with increased (IA) in the brain as the final outcome [64-66]. Age-induced IA has shown to be a consequence of the accumulation of different iron-containing molecules in different brain regions known to be particularly affected in disorders such 
as $\mathrm{AD}$ and $\mathrm{PD}[18,56]$. Cellular studies have shown that iron is specifically accumulated in microglia and astrocytes in the cerebral cortex, cerebellum, substantia nigra, and hippocampus, and it is believed that this metal ion would be involved in the neuroinflammation observed in AD and PD [28]. The mechanism underlying IA in the brain is unclear yet. However, one hypothesis holds that it is the blood brain barrier dysfunction that is responsible for the exudation of serum components, with iron among them [67]. Another hypothesis with strong experimental support proposes that IA is a consequence of the dysregulation of proteins that govern metal homeostasis. Among candidates, it has been demonstrated that the iron regulatory proteins (IREG) participate in neuronal IA. Increased expression of IREG1 has been related with neuronal survival during IA [68]. In addition, IREG2 knockout (Ireb2(-/-)) mice develop IA in white matter tracts and nuclei in different brain areas and display neurodegeneration signs in Purkinje cells [69]. Mutations in the divalent metal transporter 1 (DMT1) have been shown to impair iron transport and to protect rodents against neurotoxins like 6-hydroxydopamine, supporting a critical role for DMT1 in iron-mediated neurodegeneration [70]. Mitochondrial IA, loss of iron-sulfur cluster-containing enzymes, and increased oxidative damage are known to occur in yeast and mouse frataxin-depleted mutants as well as in tissues and cell lines from Friedrich's ataxia (FRDA) patients, suggesting that frataxin may be involved in the export of iron from the mitochondria, the synthesis of iron-sulfur clusters, and/or the protection from oxidative damage [71]. The use of Deferiprone (DFP, a chelator in clinical use for treating iron overload) in FRDA cells has been found to reduce the mitochondrial LIP increased by frataxin deficiency [72]. A new recently reported mitochondrial ferritin (MtFt) specifically expresses in high energy-consuming cells, including neurons. The overexpression of MtFt has been observed to lead to a cytosolic iron deficiency and to significantly prevent the alteration of iron redistribution and, consequently, neuronal toxicity induced by 6-hydrodopamine [69].

Although the existing data clearly show a relationship between iron metabolism, aging, and neurodegeneration, more and deeper studies are needed to completely understand the role of this transition metal in the onset and progression of neurodegenerative diseases and neurological age-related disorders.

\section{Iron Interaction with Amyloid Beta $(\mathrm{A} \beta)$ Peptide}

Interestingly, IA, as well as oxidative stress in $\mathrm{AD}$ brains, has been linked to altered $\mathrm{A} \beta$ deposition. It is well known that $\mathrm{A} \beta$ accumulates in senile plaques in $\mathrm{AD}$, and it has also been demonstrated to participate in a positive feedback loop, where oxidative stress leads to increased $\mathrm{A} \beta$ generation, and, conversely, the mechanism of $\mathrm{A} \beta$ polymerization generates oxidative stress which in turn enhances $A \beta$ production [73]. Additionally, $\mathrm{A} \beta$ has been characterized as a metalloprotein able to bind transition metals (e.g., zinc, iron, copper) via 3 histidine (positions 6, 13, and 14) and 1 tyrosine (position
10) residues located in the hydrophilic $\mathrm{N}$-terminal part of the peptide $[74,75]$, and in so doing $A \beta$ would prevent these potentially redox-active ions from causing oxidative stress. Notably, the redox potential of iron is significantly attenuated by $\mathrm{A} \beta$, supporting a neuroprotective chelating role for $\mathrm{A} \beta$ in $\mathrm{AD}$ pathogenesis $[76,77]$. This particular feature of $\mathrm{A} \beta$ could, at least in part, explain the enrichment of these transition metals in $\mathrm{AD}$ plaques [48]. It has been shown that not only ROS production induces $\mathrm{A} \beta$ aggregation but also its ability to bind metal ions as well. Augmented iron concentrations and oxidative stress have been found to correlate with changes in the concentration of both soluble and deposited $\mathrm{A} \beta$ [78]. Interestingly, the metal-dependent generation of ROS by $\mathrm{A} \beta$ may be a good target for therapeutics. For example, chelation therapy with desferrioxamine and clioquinol (which are iron and copper/zinc chelators, resp.) has shown to induce clinical improvement in patients with $\mathrm{AD}[79,80]$. Moreover, coincubation of $\mathrm{A} \beta$ from postmortem AD brains with metal chelators has shown to dissolve $\mathrm{A} \beta$ deposits [81]. In addition, both animal and human studies with clioquinol have been found to reverse $\mathrm{A} \beta$ deposition, improve cognition, general behavior and health, and lower plasma $\mathrm{A} \beta$ levels $[79,82,83]$.

Taken together, all these data clearly demonstrate that a deeper understanding of the metal-related mechanisms operating in neurodegenerative disorders such as $\mathrm{AD}$ is needed, since it may provide insights into new therapeutic approaches.

\section{Neuronal Signaling during Iron-Induced Neurotoxicity}

Neurons have developed several mechanisms in response to oxidative injury; one of them is the activation of signaling pathways that promote death or survival. The extent and duration of the oxidative insult as well as the cell type injured are crucial factors in determining which pathways are activated, their prevalence, and, in consequence, the final cellular fate $[84,85]$. In this aspect, metal-induced oxidative stress has been implicated as the triggering factor of several protective and proapoptotic signaling pathways in neurons $[7,86]$.

Synapses are sites where the first manifestations of neurodegenerative processes are likely to appear. Their vulnerability to iron-induced oxidative stress has been largely demonstrated by the presence of membrane lipid peroxidation, impairment of membrane ion-motive ATPases, glucose and glutamate transport, and mitochondrial function [87]. In this regard, several key components of signaling pathways like extracellular signal-regulated kinase (ERK), phosphoinositide 3-kinase (PI3K), Akt, and glycogen synthase kinase $3 \beta(\mathrm{GSK} 3 \beta)$ are activated in situ in isolated synaptic endings exposed to iron-induced oxidative injury $[88,89]$. Moreover, several key biochemical events that are known to occur in intact neurons undergoing apoptosis (i.e., exposure of phosphatidylserine on the plasma membrane surface, activation of caspase-3, mitochondrial calcium uptake, and ROS accumulation) also occur in isolated synaptosomes 
exposed to iron-induced oxidative stress [90]. Synapse loss, a key event in neurodegenerative disorders, might also involve in situ apoptotic cascades that might occur before, or independently of, neuronal death. This assumption is supported by the appearance of degenerative morphological changes in synapses preceding amyloid deposition and neuronal degeneration [91]. However, mechanisms whereby apoptotic events triggered by iron-induced oxidative stress in synapses propagate to the cell body remain unknown.

Some cellular signaling pathways are clearly linked to enhanced survival, while others are associated with cell death. Hence, it has been suggested that the balance between the magnitude of ERK and Jun kinase (JNK) activation is key to determining survival. While this idea is still generally accepted, more recent evidence suggests that ERK can exert apoptotic influences, and JNK can exert antiapoptotic influences during the cellular response to oxidative stress.

The presence of iron and $\mathrm{A} \beta$ provokes a marked decrease in protein kinase $\mathrm{C}$ isoforms, reduced Akt serine/threonine kinase activity, Bcl-2-associated death promoter (BAD) phosphorylation, and enhanced p38 mitogen-activated protein kinase (MAPK) and caspase- 9 and caspase- 3 activation [92-94]. In isolated synaptic endings, the coincubation with iron and $\mathrm{A} \beta$ triggers the activation of Akt and ERK signaling in an oxidation-dependent manner [95]. The phosphorylation and subsequent inhibition of GSK3 $\beta$ mediated by AMPactivated protein kinase (AMPK) contributes to protecting mitochondria against iron-catalyzed oxidative stress [96]. In $\mathrm{AD}$, abnormal activation of GSK3 $\beta$ pathway might play an important role in neurodegeneration, and compounds such as lithium that modulate GSK3 $\beta$ activity have been shown to reduce $A \beta$ production and tau phosphorylation in APP transgenic mice [97].

Deferoxamine, a known iron chelator, has been shown to block all the proapoptotic signaling events triggered by $\mathrm{A} \beta$-Fe. Moreover, $\mathrm{A} \beta$ alone has been shown not to activate proapoptotic signaling, thus demonstrating that apoptotic cell death can be only triggered by the presence of iron in vitro [92-94].

Iron has been found to be required for long-term potentiation in hippocampal CA1 neurons, and it is known to participate in the stimulation of calcium release through ROS produced via the Fenton reaction triggering the stimulation of the ERK signaling pathway. These results support a coordinated action between iron and calcium in synaptic plasticity and raise the possibility that elevated iron levels may contribute to neuronal degeneration through excessive intracellular calcium increase caused by iron-induced oxidative stress [98].

As previously mentioned, iron (alone or in combination with $\mathrm{A} \beta$ ) is able to activate different signaling cascades. The activation of these signaling pathways is, in most cases, a necessary upstream event for the activation of several transcription factors (TFs). These TFs regulate the expression of specific genes that participate in cellular events such as survival or death. Nuclear factor kappa B (NF$\kappa \mathrm{B})$ plays crucial roles in cellular resistance to oxidants and survival. Although the knowledge of NF- $\kappa \mathrm{B}$ gene targets in neurons is limited, it has been demonstrated that this TF can promote their survival and regulate synaptic plasticity [99]. The involvement of NF- $\kappa \mathrm{B}$ in the inhibition of apoptosis has been well established [100]. NF- $\kappa$ B plays a central role in the induction of neuroprotective antiapoptotic gene products, such as MnSOD and Bcl-2 that are known to contribute to ischemic tolerance [101]. Activation of NF$\kappa \mathrm{B}$ has also been associated with increased resistance of neurons to apoptosis induced by iron exposure [102]. Levels of p65 immunoreactivity have been reported to be increased in neurons and astrocytes associated with $A \beta$ plaques in the brains of $\mathrm{AD}$ patients, suggesting an increased NF- $\kappa \mathrm{B}$ activation [103]. Exposure of cultured neurons to $A \beta$ or a secreted form of amyloid precursor protein (sAPP) has shown to induce NF- $\kappa \mathrm{B}$ activation, thus suggesting a role for proteolytic products of APP in NF- $\kappa$ B activation in AD [104]. Levels of NF- $\kappa \mathrm{B}$ activity have been reported to be increased in cholinergic neurons in the basal forebrain of $\mathrm{AD}$ patients [105]. Others have established a correlation between increased NF- $\kappa \mathrm{B}$ activity and COX-2 gene transcription in brain regions affected in $\mathrm{AD}$ patients [106]. In addition, the inhibition of NF- $\kappa \mathrm{B}$ transcriptional activity results in increased vulnerability of neurons to death induced by $A \beta$ [107].

Hypoxia-inducible factor (HIF) is a TF that regulates the expression of more than 60 genes. The expression of genes relevant to iron metabolism such as ceruloplasmin, transferrin receptor, transferrin, and heme-oxygenase 1 has been shown to be regulated by HIF [108-111]. Iron chelation therapy that reduces the size of LIP has been reported to induce the activity of this TF. Moreover, a new multifunctional nontoxic, brain permeable iron chelator, M30, has shown to activate the HIF-1alpha signaling pathway in rat primary culture of cortical cells. M30 has also been found to increase the expression levels of the transcripts of brain-derived neurotrophic factor (BDNF) and growthassociated protein -43 (GAP-43). In connection with $\mathrm{AD}$, M30 has been reported to enhance the levels of phospho-Akt (Ser473) and phospho-GSK3 $\beta$ (Ser9) and to attenuate Tau phosphorylation [112].

The activator protein-1 (AP-1) is another redox-sensitive TF. AP-1 is known to participate in critical cellular processes such as proliferation, differentiation, and survival. Strong evidence supports the involvement of AP-1 in oxidative stress signaling in neurons. In rat cortical neurons and astrocytes, $\mathrm{H}_{2} \mathrm{O}_{2}$ has been demonstrated to activate MAPK cascade [113]. Upstream of AP-1, c-Jun-N-terminal kinase (JNK), and p38 (two stress-related MAPK) has also shown to be activated by increases in the intracellular levels of oxidants [114-116]. Both MAPK and AP-1 are implicated in normal physiological functions of the brain. c-Jun, a component of AP-1, has been recently attributed a dual role: it is believed to mediate neurodegeneration and cell death as well as participate in plasticity and repair mechanisms. Moreover, upregulation of iron regulatory proteins and DMT-1 isoforms after neuronal injury induced by kainate has been found to be modulated by AP- 1 in rat hippocampus [117].

Activation of JNK signaling in neurons has shown to increase stress resistance and to extend life span by the 


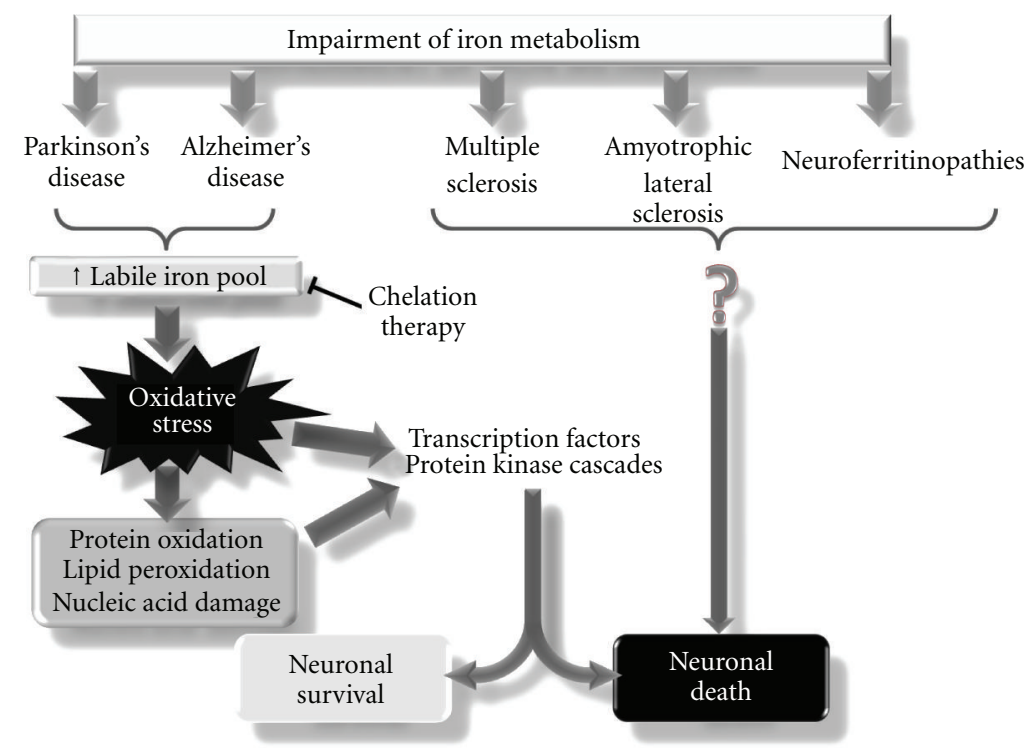

FIGURE 1: Relationship between the impairment of iron metabolism and neurodegenerative diseases. Impaired iron metabolism is a hallmark in several neurodegenerative diseases such as Parkinson's (PD) and Alzheimer's (AD) diseases, multiple sclerosis (MS), amyotrophic lateral sclerosis (ALS), and neuroferritinopathies. In the case of PD and AD, iron has been shown to play a key role in neuronal fate: depending on the extent and intensity of the oxidative stress caused by the increase in the labile iron pool, it affects transcriptional activity and signaling cascades that could participate in neuronal survival or death. Although a role for iron has also been observed in MS, ALS, and neuroferritinopathies, the molecular events that lead to neuronal death are not fully understood.

activation of the forkhead transcription factor (FOXO) family in Drosophila [118]. Recent studies have suggested that MST1 mediates oxidative stress-induced neuronal cell death by phosphorylating the transcription factor FOXO3 at serine 207, a site that is conserved in other FOXO family members $[119,120]$. All these data support the hypothesis that FOXO signaling extends life span via amelioration of oxidative damage and mitochondrial dysfunction in neurons. However, to date, there is no link between FOXO signaling and iron-induced oxidative stress in neurons. Understanding signal transduction networks that participate in iron-induced neurotoxicity constitutes one essential objective for the discovery of new drugs and treatments aimed at the improvement and delay of $\mathrm{AD}$ symptoms.

\section{Concluding Remarks}

In this paper, we summarize the latest knowledge about the role of iron in neurodegeneration processes. Iron, a redoxactive transition metal, has been proposed as an important contributing factor to the neuropathology of Alzheimer's disease. Even though increasing evidence points towards iron participation in oxidative stress events and protein aggregation, we are still far from totally comprehending the role of this transition metal in the onset and progression of neurodegenerative disorders (Figure 1). The advancement in this field will be fundamental for the establishment of new therapies intended for neuronal protection during iron mismanagement conditions.

\section{References}

[1] E. Mills, X. P. Dong, F. Wang, and H. Xu, "Mechanisms of brain iron transport: insight into neurodegeneration and CNS disorders," Future Medicinal Chemistry, vol. 2, no. 1, pp. 51-64, 2010.

[2] J. R. Connor and S. A. Benkovic, "Iron regulation in the brain: histochemical, biochemical, and molecular considerations," Annals of Neurology, vol. 32, pp. S51-S61, 1992.

[3] G. Liu, W. Huang, R. D. Moir et al., "Metal exposure and Alzheimer's pathogenesis," Journal of Structural Biology, vol. 155, no. 1, pp. 45-51, 2006.

[4] T. A. Rouault, "Systemic iron metabolism: a review and implications for brain iron metabolism," Pediatric Neurology, vol. 25, no. 2, pp. 130-137, 2001.

[5] M. B. H. Youdim, M. Fridkin, and H. Zheng, "Bifunctional drug derivatives of MAO-B inhibitor rasagiline and iron chelator VK-28 as a more effective approach to treatment of brain ageing and ageing neurodegenerative diseases," Mechanisms of Ageing and Development, vol. 126, no. 2, pp. 317-326, 2005.

[6] D. B. Kell, “Towards a unifying, systems biology understanding of large-scale cellular death and destruction caused by poorly liganded iron: Parkinson's, Huntington's, Alzheimer's, prions, bactericides, chemical toxicology and others as examples," Archives of Toxicology, vol. 84, no. 11, pp. 825-889, 2010.

[7] K. J. Barnham and A. I. Bush, "Metals in Alzheimer's and Parkinson's Diseases," Current Opinion in Chemical Biology, vol. 12, no. 2, pp. 222-228, 2008.

[8] L. Bica, P. J. Crouch, R. Cappai, and A. R. White, "Metallocomplex activation of neuroprotective signalling pathways as 
a therapeutic treatment for Alzheimer's disease," Molecular BioSystems, vol. 5, no. 2, pp. 134-142, 2009.

[9] Y. Christen, "Oxidative stress and Alzheimer disease," American Journal of Clinical Nutrition, vol. 71, no. 2, pp. 621S629S, 2000.

[10] G. Perry, L. M. Sayre, C. S. Atwood et al., "The role of iron and copper in the aetiology of neurodegenerative disorders: therapeutic implications," CNS Drugs, vol. 16, no. 5, pp. 339352, 2002.

[11] P. Zatta, R. Lucchini, S. J. Van Rensburg, and A. Taylor, "The role of metals in neurodegenerative processes: aluminum, manganese, and zinc," Brain Research Bulletin, vol. 62, no. 1, pp. 15-28, 2003.

[12] H. -H. Cho, C. M. Cahill, C. R. Vanderburg et al., "Selective translational control of the Alzheimer amyloid precursor protein transcript by iron regulatory protein-1," Journal of Biological Chemistry, vol. 285, no. 41, pp. 31217-31232, 2010.

[13] J. Frackowiak, A. Potempska, and B. Mazur-Kolecka, "Formation of amyloid- $\beta$ oligomers in brain vascular smooth muscle cells transiently exposed to iron-induced oxidative stress," Acta Neuropathologica, vol. 117, no. 5, pp. 557-567, 2009.

[14] D. R. Richardson and P. Ponka, "The molecular mechanisms of the metabolism and transport of iron in normal and neoplastic cells," Biochimica et Biophysica Acta, vol. 1331, no. 1, pp. 1-40, 1997.

[15] B. Halliwell, "The wanderings of a free radical," Free Radical Biology and Medicine, vol. 46, no. 5, pp. 531-542, 2009.

[16] B. Halliwell, "Role of free radicals in the neurodegenerative diseases: therapeutic implications for antioxidant treatment," Drugs and Aging, vol. 18, no. 9, pp. 685-716, 2001.

[17] Y. A. Ke and Z. M. Qian, "Brain iron metabolism: neurobiology and neurochemistry," Progress in Neurobiology, vol. 83, no. 3, pp. 149-173, 2007.

[18] L. Zecca, M. B. H. Youdim, P. Riederer, J. R. Connor, and R. R. Crichton, "Iron, brain ageing and neurodegenerative disorders," Nature Reviews Neuroscience, vol. 5, no. 11, pp. 863-873, 2004.

[19] Y. J. Suh and S. David, "Age-related changes in iron homeostasis and cell death in the cerebellum of ceruloplasmindeficient mice," Journal of Neuroscience, vol. 26, no. 38, pp. 9810-9819, 2006.

[20] S. J. Texel, X. Xu, and Z. L. Harris, "Ceruloplasmin in neurodegenerative diseases," Biochemical Society Transactions, vol. 36, part 6, pp. 1277-1281, 2008.

[21] A. V. Alekseenko, T. V. Waseem, and S. V. Fedorovich, "Ferritin, a protein containing iron nanoparticles, induces reactive oxygen species formation and inhibits glutamate uptake in rat brain synaptosomes," Brain Research, vol. 1241, pp. 193-200, 2008.

[22] P. F. Chinnery, D. E. Crompton, D. Birchall et al., "Clinical features and natural history of neuroferritinopathy caused by the FTL1 460InsA mutation," Brain, vol. 130, no. 1, pp. 110119, 2007.

[23] O. Gakh, T. Bedekovics, S. F. Duncan, D. Y. Smith, D. S. Berkholz, and G. Isaya, "Normal and Friedreich ataxia cells express different isoforms of frataxin with complementary roles in iron-sulfur cluster assembly," Journal of Biological Chemistry. In press.

[24] M. C. Mantovan, A. Martinuzzi, F. Squarzanti et al., "Exploring mental status in Friedreich's ataxia: a combined neuropsychological, behavioral and neuroimaging study," European Journal of Neurology, vol. 13, no. 8, pp. 827-835, 2006.

[25] D. Berg and M. B. Youdim, "Role of iron in neurodegenerative disorders," Topics in Magnetic Resonance Imaging, vol. 17, no. 1, pp. 5-17, 2006.

[26] D. T. Dexter, P. Jenner, A. H.V. Schapira, and C. D. Marsden, "Alterations in levels of iron, ferritin, and other trace metals in neurodegenerative diseases affecting the basal ganglia. The Royal Kings and Queens Parkinson's Disease Research Group," Annals of Neurology, vol. 32, supplement, pp. S94S100, 1992.

[27] D. A. Simmons, M. Casale, B. Alcon, N. Pham, N. Narayan, and G. Lynch, "Ferritin accumulation in dystrophic microglia is an early event in the development of Huntington's Disease," GLIA, vol. 55, no. 10, pp. 1074-1084, 2007.

[28] W. Y. Ong and A. A. Farooqui, "Iron, neuroinflammation, and Alzheimer's disease," Journal of Alzheimer's Disease, vol. 8, no. 2, pp. 183-200, 2005.

[29] C. Quintana, S. Bellefqih, J. Y. Laval et al., "Study of the localization of iron, ferritin, and hemosiderin in Alzheimer's disease hippocampus by analytical microscopy at the subcellular level," Journal of Structural Biology, vol. 153, no. 1, pp. 42-54, 2006.

[30] T. A. Rouault, "Iron on the brain," Nature Genetics, vol. 28, no. 4, pp. 299-300, 2001.

[31] C. N. Roy and N. C. Andrews, "Recent advances in disorders of iron metabolism: mutations, mechanisms and modifiers," Human Molecular Genetics, vol. 10, no. 20, pp. 2181-2186, 2001.

[32] Y. Imon, S. Yamaguchi, Y. Yamamura et al., "Low intensity areas observed on T2-weighted magnetic resonance imaging of the cerebral cortex in various neurological diseases," Journal of the Neurological Sciences, vol. 134, supplement 1, pp. 27-32, 1995.

[33] J. Burn and P. F. Chinnery, "Neuroferritinopathy," Seminars in Pediatric Neurology, vol. 13, no. 3, pp. 176-181, 2006.

[34] E. J. Kasarskis, L. Tandon, M. A. Lovell, and W. D. Ehmann, "Aluminum, calcium, and iron in the spinal cord of patients with sporadic amyotrophic lateral sclerosis using laser microprobe mass spectroscopy: a preliminary study," Journal of the Neurological Sciences, vol. 130, no. 2, pp. 203-208, 1995.

[35] H. Oba, T. Araki, K. Ohtomo et al., "Amyotrophic lateral sclerosis: T2 shortening in motor cortex at MR imaging," Radiology, vol. 189, no. 3, pp. 843-846, 1993.

[36] A. Gregory and S. J. Hayflick, "Neurodegeneration with brain iron accumulation," Folia Neuropathologica, vol. 43, no. 4, pp. 286-296, 2005.

[37] S. Levi, A. Cozzi, and P. Arosio, "Neuroferritinopathy: a neurodegenerative disorder associated with L-ferritin mutation," Best Practice and Research: Clinical Haematology, vol. 18, no. 2, pp. 265-276, 2005.

[38] A. R. J. Curtis, C. Fey, C. M. Morris et al., "Mutation in the gene encoding ferritin light polypeptide causes dominant adult-onset basal ganglia disease," Nature Genetics, vol. 28, no. 4, pp. 350-354, 2001.

[39] S. M. Levine, "Iron deposits in multiple sclerosis and Alzheimer's disease brains," Brain Research, vol. 760, no. 12, pp. 298-303, 1997.

[40] S. M. LeVine and A. Chakrabarty, "The role of iron in the pathogenesis of experimental allergic encephalomyelitis 
and multiple sclerosis," Annals of the New York Academy of Sciences, vol. 1012, pp. 252-266, 2004.

[41] A. Chakrabarty, M. R. Emerson, and S. M. LeVine, "Heme oxygenase-I in SJL mice with experimental allergic encephalomyelitis," Multiple Sclerosis, vol. 9, no. 4, pp. 372381, 2003.

[42] S. M. LeVine, S. G. Lynch, C. -N. Ou, M. J. Wulser, E. Tam, and N. Boo, "Ferritin, transferrin and iron concentrations in the cerebrospinal fluid of multiple sclerosis patients," Brain Research, vol. 821, no. 2, pp. 511-515, 1999.

[43] C. Sfagos, A. C. Makis, A. Chaidos et al., "Serum ferritin, transferrin and soluble transferrin receptor levels in multiple sclerosis patients," Multiple Sclerosis, vol. 11, no. 3, pp. 272275, 2005.

[44] L. M. Sayre, D. A. Zelasko, P. L. R. Harris, G. Perry, R. G. Salomon, and M. A. Smith, "4-Hydroxynonenal-derived advanced lipid peroxidation end products are increased in Alzheimer's disease," Journal of Neurochemistry, vol. 68, no. 5, pp. 2092-2097, 1997.

[45] M. A. Smith, M. Tabaton, and G. Perry, "Early contribution of oxidative glycation in Alzheimer disease [2]," Neuroscience Letters, vol. 217, no. 2-3, pp. 210-211, 1996.

[46] M. A. Smith, P. L. R. Harris, L. M. Sayre, and G. Perry, "Iron accumulation in Alzheimer disease is a source of redoxgenerated free radicals," Proceedings of the National Academy of Sciences of the United States of America, vol. 94, no. 18, pp. 9866-9868, 1997.

[47] M. L. Hegde, P. M. Hegde, L. M.F. Holthauzen, T. K. Hazra, K. S.J. Rao, and S. Mitra, "Specific inhibition of NEIL-initiated repair of oxidized base damage in human genome by copper and iron: potential etiological linkage to neurodegenerative diseases," Journal of Biological Chemistry, vol. 285, no. 37, pp. 28812-28825, 2010.

[48] M. A. Lovell, J. D. Robertson, W. J. Teesdale, J. L. Campbell, and W. R. Markesbery, "Copper, iron and zinc in Alzheimer's disease senile plaques," Journal of the Neurological Sciences, vol. 158, no. 1, pp. 47-52, 1998.

[49] M. F. Falangola, S. -P. Lee, R. A. Nixon, K. Duff, and J. A. Helpern, "Histological co-localization of iron in $\mathrm{A} \beta$ plaques of PS/APP transgenic mice," Neurochemical Research, vol. 30, no. 2, pp. 201-205, 2005.

[50] R. A. Cherny, J. T. Legg, C. A. McLean et al., "Aqueous dissolution of Alzheimer's disease $\mathrm{A} \beta$ amyloid deposits by biometal depletion," Journal of Biological Chemistry, vol. 274, no. 33, pp. 23223-23228, 1999.

[51] D. A. Butterfield, M. L. Bader Lange, and R. Sultana, "Involvements of the lipid peroxidation product, HNE, in the pathogenesis and progression of Alzheimer's disease," Biochimica et Biophysica Acta, vol. 1801, no. 8, pp. 924-929, 2010.

[52] M. A. Smith, X. Zhu, M. Tabaton et al., "Increased iron and free radical generation in preclinical Alzheimer disease and mild cognitive impairment," Journal of Alzheimer's Disease, vol. 19, no. 1, pp. 363-372, 2010.

[53] W. -Z. Zhu, W. -D. Zhong, W. Wang et al., "Quantitative MR phase-corrected imaging to investigate increased brain iron deposition of patients with Alzheimer disease," Radiology, vol. 253, no. 2, pp. 497-504, 2009.

[54] E. V. Sullivan, E. Adalsteinsson, T. Rohlfing, and A. Pfefferbaum, "Relevance of iron deposition in deep gray matter brain structures to cognitive and motor performance in healthy elderly men and women: exploratory findings," Brain Imaging and Behavior, vol. 3, no. 2, pp. 167-175, 2009.
[55] J. F. Collingwood, A. Mikhaylova, M. Davidson et al., "In situ characterization and mapping of iron compounds in Alzheimer's disease tissue," Journal of Alzheimer's Disease, vol. 7, no. 4, pp. 267-272, 2005.

[56] G. Bartzokis, T. A. Tishler, IL. S. Shin, PO. H. Lu, and J. L. Cummings, "Brain ferritin iron as a risk factor for age at onset in neurodegenerative diseases," Annals of the New York Academy of Sciences, vol. 1012, pp. 224-236, 2004.

[57] W. A. Cass, R. Grondin, A. H. Andersen et al., "Iron accumulation in the striatum predicts aging-related decline in motor function in rhesus monkeys," Neurobiology of Aging, vol. 28, no. 2, pp. 258-271, 2007.

[58] J. R. Connor, B. S. Snyder, J. L. Beard, R. E. Fine, and E. J. Mufson, "Regional distribution of iron and iron-regulatory proteins in the brain in aging and Alzheimer's disease," Journal of Neuroscience Research, vol. 31, no. 2, pp. 327-335, 1992.

[59] W. R. W. Martin, F. Q. Ye, and P. S. Allen, "Increasing striatal iron content associated with normal aging," Movement Disorders, vol. 13, no. 2, pp. 281-286, 1998.

[60] G. Casadesus, M. A. Smith, X. Zhu et al., "Alzheimer disease: evidence for a central pathogenic role of iron-mediated reactive oxygen species," Journal of Alzheimer's Disease, vol. 6, no. 2, pp. 165-169, 2004.

[61] D. Kaur and J. Andersen, "Does cellular iron dysregulation play a causative role in Parkinson's disease?" Ageing Research Reviews, vol. 3, no. 3, pp. 327-343, 2004.

[62] YA. Ke and Z. M. Qian, "Iron misregulation in the brain: a primary cause of neurodegenerative disorders," Lancet Neurology, vol. 2, no. 4, pp. 246-253, 2003.

[63] M. Thomas and J. Jankovic, "Neurodegenerative disease and iron storage in the brain," Current Opinion in Neurology, vol. 17, no. 4, pp. 437-442, 2004.

[64] G. Bartzokis, P. H. Lu, T. A. Tishler et al., "Prevalent iron metabolism gene variants associated with increased brain ferritin iron in healthy older men," Journal of Alzheimer's Disease, vol. 20, no. 1, pp. 333-341, 2010.

[65] A. S. Dornelles, V. A. Garcia, M. N. M. De Lima et al., "mRNA expression of proteins involved in iron homeostasis in brain regions is altered by age and by iron overloading in the neonatal period," Neurochemical Research, vol. 35, no. 4, pp. 564-571, 2010.

[66] X. Xu, Q. Wang, and M. Zhang, "Age, gender, and hemispheric differences in iron deposition in the human brain: an in vivo MRI study," NeuroImage, vol. 40, no. 1, pp. 35-42, 2008.

[67] T. Morita, Y. Mizutani, M. Sawada, and A. Shimada, "Immunohistochemical and ultrastructural findings related to the blood-brain barrier in the blood vessels of the cerebral white matter in aged dogs," Journal of Comparative Pathology, vol. 133, no. 1, pp. 14-22, 2005.

[68] P. Aguirre, N. Mena, V. Tapia, M. Arredondo, and M. T. Núñez, "Iron homeostasis in neuronal cells: a role for IREG1,” BMC Neuroscience, vol. 6, article 3, 2005.

[69] Z. -H. Shi, G. Nie, X. -L. Duan et al., "Neuroprotective mechanism of mitochondrial ferritin on 6-hydroxydopamineinduced dopaminergic cell damage: implication for neuroprotection in Parkinson's disease," Antioxidants and Redox Signaling, vol. 13, no. 6, pp. 783-796, 2010.

[70] J. Salazar, N. Mena, S. Hunot et al., "Divalent metal transporter 1 (DMT1) contributes to neurodegeneration in animal models of Parkinson's disease," Proceedings of the 
National Academy of Sciences of the United States of America, vol. 105, no. 47, pp. 18578-18583, 2008.

[71] H. Puccio, D. Simon, M. Cossée et al., "Mouse models for Friedreich ataxia exhibit cardiomyopathy, sensory nerve defect and Fe-S enzyme deficiency followed by intramitochondrial iron deposits," Nature Genetics, vol. 27, no. 2, pp. 181-186, 2001.

[72] OR. Kakhlon, W. Breuer, A. Munnich, and Z. I. Cabantchik, "Iron redistribution as a therapeutic strategy for treating diseases of localized iron accumulation," Canadian Journal of Physiology and Pharmacology, vol. 88, no. 3, pp. 187-196, 2010.

[73] L. Zhang, B. Zhao, D. T. Yew, J. W. Kusiak, and G. S. Roth, "Processing of Alzheimer's amyloid precursor protein during HO-induced apoptosis in human neuronal cells," Biochemical and Biophysical Research Communications, vol. 235, no. 3, pp. 845-848, 1997.

[74] C. S. Atwood, R. D. Moir, X. Huang et al., "Dramatic aggregation of alzheimer by $\mathrm{Cu}$ (II) is induced by conditions representing physiological acidosis," Journal of Biological Chemistry, vol. 273, no. 21, pp. 12817-12826, 1998.

[75] C. S. Atwood, R. C. Scarpa, X. Huang et al., "Characterization of copper interactions with Alzheimer amyloid $\beta$ peptides: identification of an attomolar-affinity copper binding site on amyloid $\beta 1-42$," Journal of Neurochemistry, vol. 75, no. 3, pp. 1219-1233, 2000.

[76] C. A. Rottkamp, C. S. Atwood, J. A. Joseph, A. Nunomura, G. Perry, and M. A. Smith, "The state versus amyloid- $\beta$ : the trial of the most wanted criminal in Alzheimer disease," Peptides, vol. 23, no. 7, pp. 1333-1341, 2002.

[77] L. M. Sayre, G. Perry, P. L. R. Harris, Y. Liu, K. A. Schubert, and M. A. Smith, "In situ oxidative catalysis by neurofibrillary tangles and senile plaques in Alzheimer's disease: a central role for bound transition metals," Journal of Neurochemistry, vol. 74, no. 1, pp. 270-279, 2000.

[78] C. S. Atwood, M. E. Obrenovich, T. Liu et al., "Amyloid- $\beta$ : a chameleon walking in two worlds: a review of the trophic and toxic properties of amyloid- $\beta$," Brain Research Reviews, vol. 43, no. 1, pp. 1-16, 2003.

[79] C. W. Ritchie, A. I. Bush, A. Mackinnon et al., "Metalprotein attenuation with Iodochlorhydroxyquin (Clioquinol) targeting $\mathrm{A} \beta$ amyloid deposition and toxicity in alzheimer disease: a pilot phase 2 clinical trial," Archives of Neurology, vol. 60, no. 12, pp. 1685-1691, 2003.

[80] C. A. Perez, Y. Tong, and M. Guo, "Iron chelators as potential therapeutic agents for Parkinson's disease," Current Bioactive Compounds, vol. 4, no. 3, pp. 150-158, 2008.

[81] R. A. Cherny, K. J. Barnham, T. Lynch et al., "Chelation and intercalation: complementary properties in a compound for the treatment of Alzheimer's disease," Journal of Structural Biology, vol. 130, no. 2-3, pp. 209-216, 2000.

[82] R. A. Cherny, C. S. Atwood, M. E. Xilinas et al., "Treatment with a copper-zinc chelator markedly and rapidly inhibits $\beta$-amyloid accumulation in Alzheimer's disease transgenic mice," Neuron, vol. 30, no. 3, pp. 665-676, 2001.

[83] B. Regland, W. Lehmann, I. Abedini et al., "Treatment of Alzheimer's disease with clioquinol," Dementia and Geriatric Cognitive Disorders, vol. 12, no. 6, pp. 408-414, 2001.

[84] A. Nunomura, P. I. Moreira, H. G. Lee et al., "Neuronal death and survival under oxidative stress in Alzheimer and Parkinson diseases," CNS and Neurological Disorders-Drug Targets, vol. 6, no. 6, pp. 411-423, 2007.
[85] R. B. Petersen, A. Nunomura, H. G. Lee et al., "Signal transduction cascades associated with oxidative stress in Alzheimer's disease," Journal of Alzheimer's Disease, vol. 11, no. 2, pp. 143-152, 2007.

[86] G. A. Salvador, "Iron in neuronal function and dysfunction," BioFactors, vol. 36, no. 2, pp. 103-110, 2010.

[87] Z. H. Guo and M. P. Mattson, "Neurotrophic factors protect cortical synaptic terminals against amyloid- and oxidative stress-induced impairment of glucose transport, glutamate transport and mitochondrial function," Cerebral Cortex, vol. 10, no. 1, pp. 50-57, 2000.

[88] R. M. Uranga, M. V. Mateos, N. M. Giusto, and G. A. Salvador, "Activation of phosphoinositide-3 kinase/Akt pathway by $\mathrm{FeSO}_{4}$ in rat cerebral cortex synaptic endings," Journal of Neuroscience Research, vol. 85, no. 13, pp. 2924-2932, 2007.

[89] R. M. Uranga, N. M. Giusto, and G. A. Salvador, "Ironinduced oxidative injury differentially regulates PI3K/Akt/ GSK3 $\beta$ pathway in synaptic endings from adult and aged rats," Toxicological Sciences, vol. 111, no. 2, pp. 331-344, 2009.

[90] M. P. Mattson, "Apoptotic and anti-apoptotic synaptic signaling mechanisms," Brain Pathology, vol. 10, no. 2, pp. 300-312, 2000.

[91] S. T. DeKosky, S. W. Scheff, and S. D. Styren, "Structural correlates of cognition in dementia: quantification and assessment of synapse change," Neurodegeneration, vol. 5, no. 4, pp. 417-421, 1996.

[92] F. Kuperstein and E. Yavin, "Pro-apoptotic signaling in neuronal cells following iron and amyloid beta peptide neurotoxicity," Journal of Neurochemistry, vol. 86, no. 1, pp. 114-125, 2003.

[93] F. Kuperstein, A. Brand, and E. Yavin, "Amyloid A $\beta$ preconditions non-apoptotic signals in vivo and protects fetal rat brain from intrauterine ischemic stress," Journal of Neurochemistry, vol. 91, no. 4, pp. 965-974, 2004.

[94] M. P. Mattson, "NF- $\kappa$ B in the survival and plasticity of neurons," Neurochemical Research, vol. 30, no. 6-7, pp. 883893, 2005.

[95] R. M. Uranga, N. M. Giusto, and G. A. Salvador, "Effect of transition metals in synaptic damage induced by amyloid beta peptide," Neuroscience, vol. 170, no. 2, pp. 381-389, 2010.

[96] S. H. Choi, Y. W. Kim, and S. G. Kim, "AMPK-mediated GSK3 $\beta$ inhibition by isoliquiritigenin contributes to protecting mitochondria against iron-catalyzed oxidative stress," Biochemical Pharmacology, vol. 79, no. 9, pp. 1352-1362, 2010.

[97] E. Rockenstein, M. Torrance, A. Adame et al., "Neuroprotective effects of regulators of the glycogen synthase kinase- $3 \beta$ signaling pathway in a transgenic model of Alzheimer's disease are associated with reduced amyloid precursor protein phosphorylation," Journal of Neuroscience, vol. 27, no. 8, pp. 1981-1991, 2007.

[98] C. Hidalgo and M. T. Núñez, "Calcium, iron and neuronal function," IUBMB Life, vol. 59, no. 4-5, pp. 280-285, 2007.

[99] A. M. Adamo and P. I. Oteiza, "Zinc deficiency and neurodevelopment: the case of neurons," BioFactors, vol. 36, no. 2, pp. 117-124, 2010.

[100] M. P. Mattson, C. Culmsee, Z. Yu, and S. Camandola, "Roles of nuclear factor $\kappa \mathrm{B}$ in neuronal survival and plasticity," Journal of Neurochemistry, vol. 74, no. 2, pp. 443-456, 2000.

[101] T. Toyoda, N. F. Kassell, and K. S. Lee, "Induction of ischemic tolerance and antioxidant activity by brief focal ischemia," NeuroReport, vol. 8, no. 4, pp. 847-851, 1997. 
[102] G. W. Glazner, S. Camandola, and M. P. Mattson, "Nuclear factor- $\kappa \mathrm{B}$ mediates the cell survival-promoting action of activity-dependent neurotrophic factor peptide-9," Journal of Neurochemistry, vol. 75, no. 1, pp. 101-108, 2000.

[103] K. Terai, A. Matsuo, and P. L. McGeer, "Enhancement of immunoreactivity for NF- $\kappa \mathrm{B}$ in the hippocampal formation and cerebral cortex of Alzheimer's disease," Brain Research, vol. 735, no. 1, pp. 159-168, 1996.

[104] K. Z. Bourne, D. C. Ferrari, C. Lange-Dohna, S. Roßner, T. G. Wood, and J. R. Perez-Polo, "Differential regulation of BACE1 promoter activity by nuclear factor- $\kappa \mathrm{B}$ in neurons and glia upon exposure to $\beta$-amyloid peptides," Journal of Neuroscience Research, vol. 85, no. 6, pp. 1194-1204, 2007.

[105] F. Boissière, S. Hunot, B. Faucheux et al., "Nuclear translocation of NF- $\kappa \mathrm{B}$ in cholinergic neurons of patients with Alzheimer's disease," NeuroReport, vol. 8, no. 13, pp. 28492852, 1997.

[106] W. J. Lukiw and N. G. Bazan, "Strong nuclear factor- $\kappa$ B-DNA binding parallels cyclooxygenase-2 gene transcription in aging and in sporadic Alzheimer's disease superior temporal lobe neocortex," Journal of Neuroscience Research, vol. 53, no. 5, pp. 583-592, 1998.

[107] B. Kaltschmidt, M. Uherek, H. Wellmann, B. Volk, and C. Kaltschmidt, "Inhibition of NF- $\kappa \mathrm{B}$ potentiates amyloid $\beta$ mediated neuronal apoptosis," Proceedings of the National Academy of Sciences of the United States of America, vol. 96, no. 16, pp. 9409-9414, 1999.

[108] D. W. Lee and J. K. Andersen, "Role of HIF-1 in iron regulation: potential therapeutic strategy for neurodegenerative disorders," Current Molecular Medicine, vol. 6, no. 8, pp. 883893, 2006.

[109] F. Martin, T. Linden, D. M. Katschinski et al., "Copperdependent activation of hypoxia-inducible factor (HIF)-1: implications for ceruloplasmin regulation," Blood, vol. 105, no. 12, pp. 4613-4619, 2005.

[110] L. Tacchini, E. Gammella, C. De Ponti, S. Recalcati, and G. Cairo, "Role of HIF-1 and NF- $\kappa$ B transcription factors in the modulation of transferrin receptor by inflammatory and anti-inflammatory signals," Journal of Biological Chemistry, vol. 283, no. 30, pp. 20674-20686, 2008.

[111] D. Wang, L. -H. Wang, Y. Zhao, Y. -P. Lu, and L. Zhu, "Hypoxia regulates the ferrous iron uptake and reactive oxygen species level via divalent metal transporter 1 (DMT1) exon1B by hypoxia-inducible factor-1," IUBMB Life, vol. 62, no. 8, pp. 629-636, 2010.

[112] Y. Avramovich-Tirosh, O. Bar-Am, T. Amit, M. B.H. Youdim, and O. Weinreb, "Up-regulation of hypoxia-inducible factor (hif)- $1 \alpha$ and hif-target genes in cortical neurons by the novel multifunctional iron chelator anti-alzheimer drug, M30," Current Alzheimer Research, vol. 7, no. 4, pp. 300-306, 2010.

[113] M. Scortegagna, Z. Galdzicki, S. I. Rapoport, and I. Hanbauer, "Activator protein-1 DNA binding activation by hydrogen peroxide in neuronal and astrocytic primary cultures of trisomy-16 and diploid mice," Molecular Brain Research, vol. 73, no. 1-2, pp. 144-150, 1999.

[114] L. Aimo and P. I. Oteiza, "Zinc deficiency increases the susceptibility of human neuroblastoma cells to lead-induced activator protein-1 activation," Toxicological Sciences, vol. 91, no. 1, pp. 184-191, 2006.

[115] W. M. Toone, B. A. Morgan, and N. Jones, "Redox control of AP-1-like factors in yeast and beyond," Oncogene, vol. 20, no. 19, pp. 2336-2346, 2001.
[116] L. Benvenisti-Zarom, J. Chen-Roetling, and R. F. Regan, "Inhibition of the ERK/MAP kinase pathway attenuates heme oxygenase- 1 expression and heme-mediated neuronal injury," Neuroscience Letters, vol. 398, no. 3, pp. 230-234, 2006.

[117] E. Huang, W. -Y. Ong, M. -L. Go, and J. R. Connor, "Upregulation of iron regulatory proteins and divalent metal transporter-1 isoforms in the rat hippocampus after kainate induced neuronal injury," Experimental Brain Research, vol. 170, no. 3, pp. 376-386, 2006.

[118] K. S. Lee, K. Iijima-Ando, K. Iijima et al., "JNK/FOXOmediated neuronal expression of fly homologue of peroxiredoxin II reduces oxidative stress and extends life span," Journal of Biological Chemistry, vol. 284, no. 43, pp. 2945429461, 2009.

[119] M. K. Lehtinen, Z. Yuan, P. R. Boag et al., "A Conserved MST-FOXO Signaling Pathway Mediates Oxidative-Stress Responses and Extends Life Span," Cell, vol. 125, no. 5, pp. 987-1001, 2006.

[120] Z. Yuan, M. K. Lehtinen, P. Merlo, J. Villén, S. Gygi, and A. Bonni, "Regulation of neuronal cell death by MST1-FOXO1 signaling," Journal of Biological Chemistry, vol. 284, no. 17, pp. 11285-11292, 2009. 


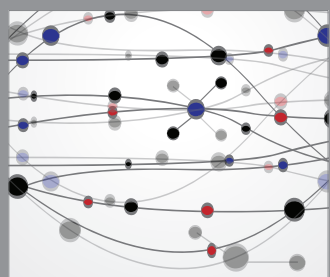

The Scientific World Journal
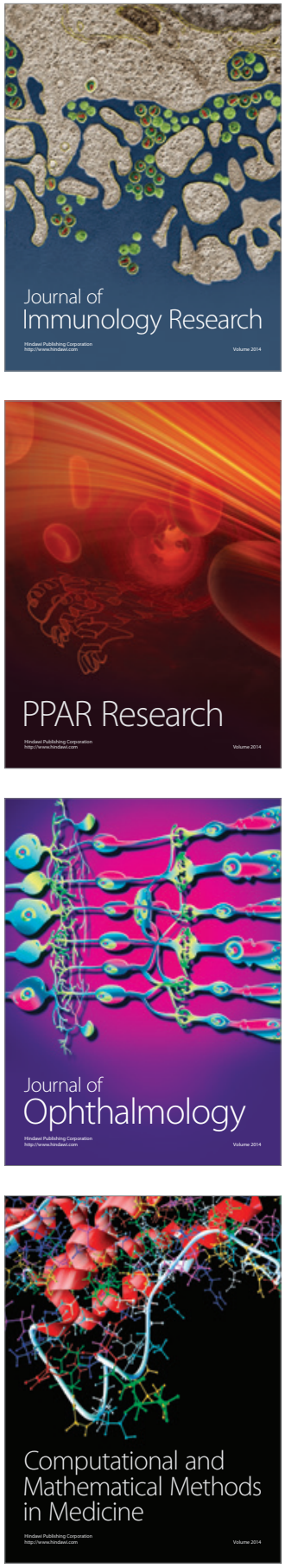

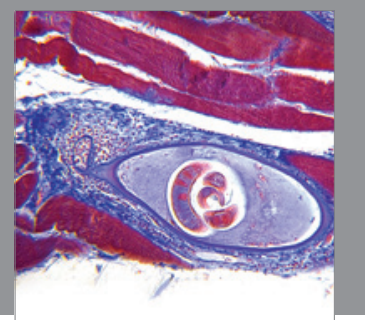

Gastroenterology

Research and Practice
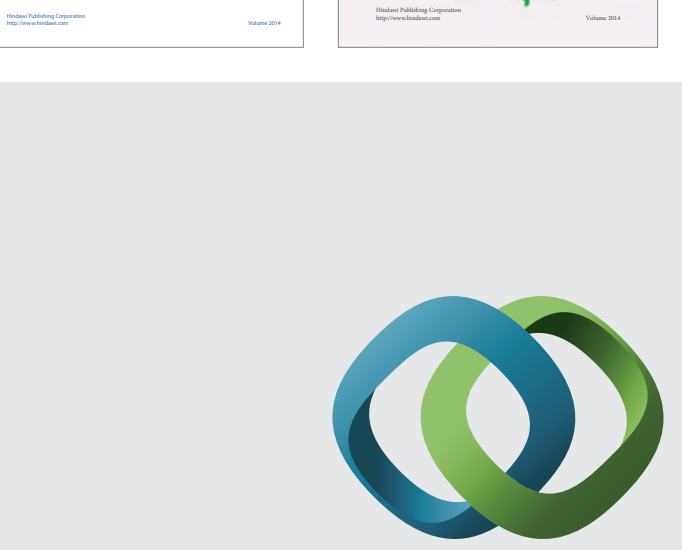

\section{Hindawi}

Submit your manuscripts at

http://www.hindawi.com
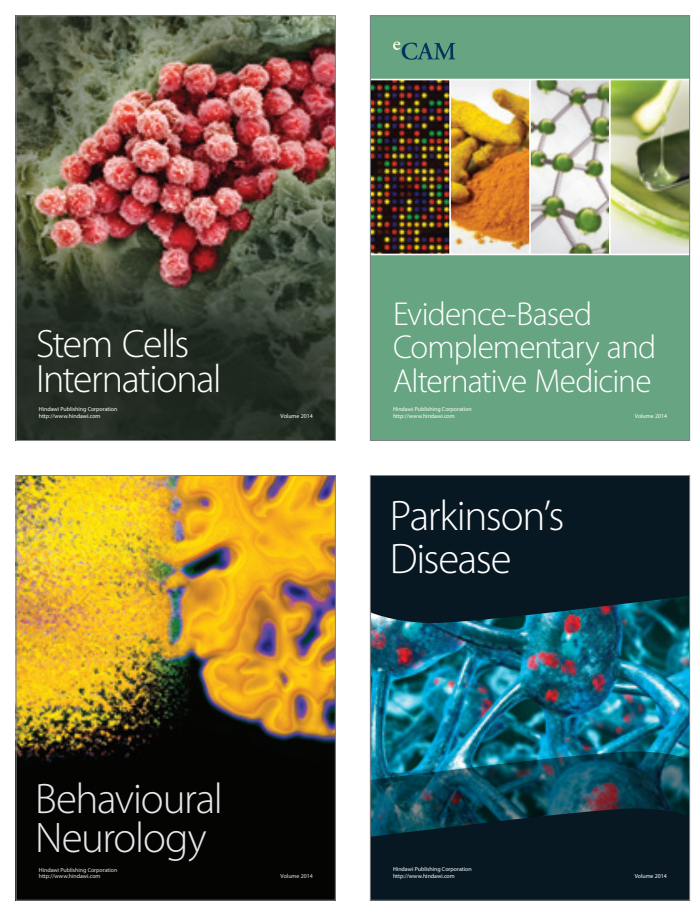

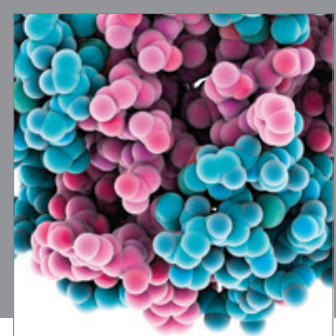

Journal of
Diabetes Research

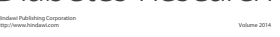

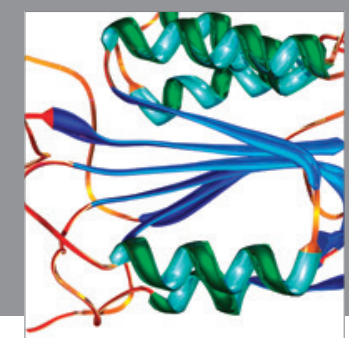

Disease Markers
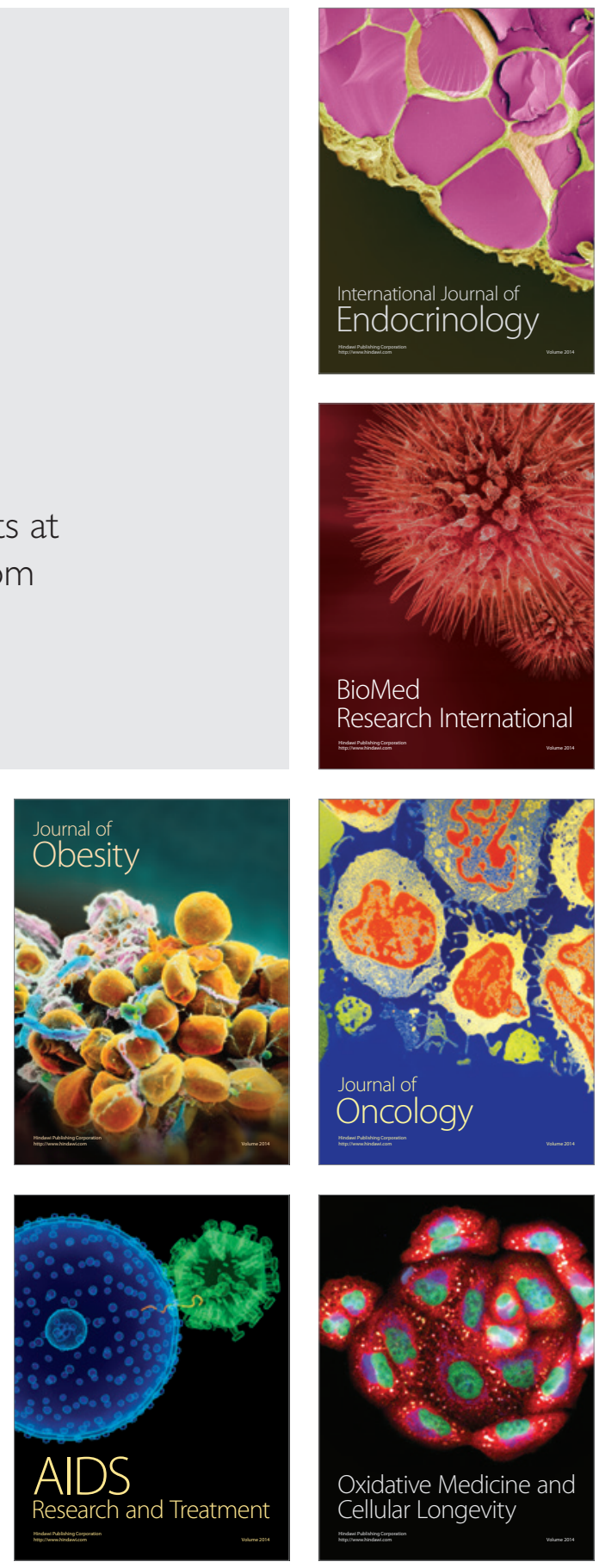